\title{
Appraisal of short and long versions of the Nutrition Environment Measures Survey (NEMS-S and NEMS-R) in Australia
}

\author{
Julia Elizabeth Carins ${ }^{1,2, *}$, Sharyn Rundle-Thiele ${ }^{1}$ and Ryan James Storr ${ }^{1}$ \\ 'Social Marketing @ Griffith, Department of Marketing, Griffith University, 170 Kessels Road, Nathan, QLD 4111, \\ Australia: ${ }^{2}$ Food \& Nutrition Group, Land Division, Defence Science and Technology Group, Scottsdale, TAS, \\ Australia
}

Submitted 22 March 2018: Final revision received 14 August 2018: Accepted 13 September 2018: First published online 30 October 2018

\begin{abstract}
Objective: Research has begun to take a more ecological view of eating behaviour, examining multiple levels of influence: personal, social and environmental. The food environment is a major influence on eating behaviour, attracting the attention of researchers who have measured it in a number of ways. The present paper examines the short-form version, in comparison to the long-form version, of the Nutrition Environment Measures Survey (NEMS) - an observational food outlet audit tool.

Design: Both the short-form and long-form were examined to qualitatively appraise the dimensions of the food environment assessed by each measure. Data from 135 food outlets in Australia were then used to compare results obtained using the short-form with the results from the long-form method, to consider the utility of the short-form measure.

Setting: The retail food environment in Australia.

Participants: One hundred and thirty-five food outlets in Australia.

Results: Results indicate that the short-form predominantly assessed availability of healthful foods (one aspect of the food environment). Several critical dimensions of the food environment known to influence eating behaviour were not assessed. For this data set, the short-form produced scores inconsistent with the longer version of the measure, delivering inflated estimates for stores and deflated estimates for restaurants.

Conclusions: Scores between the long-form and short-form versions were not comparable in this Australian study. Further development of food environment measures is recommended and must balance instrument brevity with the need to accurately capture important aspects of the food environment known to influence eating behaviour.
\end{abstract}

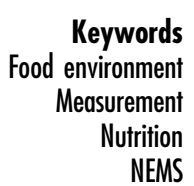

The prevalence of obesity and dietary-related non-communicable diseases is increasing, fuelled by energy-dense but nutrient-poor diets and more sedentary lifestyles ${ }^{(1,2)}$. Given the food environment is a major influence on $\operatorname{diet}^{(3)}$, measuring the food or nutrition environment is an important objective for researchers to inform the development of both individual and environmental strategies to increase healthful eating.

Defining the food environment and determining how to measure it is a complicated task ${ }^{(4)}$. The food environment encompasses a number of levels: the community level (e.g. the distribution of food sources and types within a suburb), the social level (e.g. the characteristics of the home, school or workplace) and the consumer level (e.g. aspects that the consumer experiences within an outlet) ${ }^{(5)}$. The current study focuses on the consumer level, where a number of dimensions reduce or increase opportunities to eat healthfully. These include access, availability, affordability, quality, price, promotion, placement, variety, freshness, information, promotion, media and advertising ${ }^{(5-7)}$.

The food environment has been measured in many ways $^{(4,8)}$; at least forty-eight different food environment instruments have been used ${ }^{(5)}$, eighteen of which focused 
on measuring the consumer environment (in outlets such as stores, restaurants) wholly or as part of the instrument. Of these, the Nutrition Environment Measures Survey for Stores (NEMS-S) and Nutrition Environment Measures Survey for Restaurants (NEMS-R) are the most commonly used measures of the retail food environment ${ }^{(9)}$. NEMS measures have been used in many countries and various contexts to explore the food environment in rural ${ }^{(10)}$ and urban areas ${ }^{(11)}$; the food environment that surrounds disadvantaged populations $^{(12)}$; medical ${ }^{(13)}$, educational ${ }^{(14,15)}$ and recreational facilities ${ }^{(16)}$; and indigenous ${ }^{(17)}$ and cultural groups ${ }^{(18)}$. Both instruments are observational checklists - the NEMS-S measuring aspects of availability, placement, quality and pricing in stores, and the NEMS-R measuring aspects of availability, information, promotion, facilitators, barriers and pricing in restaurants - to understand the level of support for healthful eating.

The NEMS-S and NEMS-R observational instruments can be considered time-consuming to administer, taking between 14 and $42 \mathrm{~min}$ per outlet (based on the original research studies $)^{(19,20)}$. Acknowledging that time to administer equates to a dollar cost for research, examining short-form measurements represents a worthy research endeavour. Partington et al. ${ }^{(21)}$ used data mining techniques on data from 820 stores and 1795 restaurants in the USA to select the items most predictive of the final NEMS scores, to produce short-form instruments. The short-form instrument retained only the most predictive items, weighting these in a regression equation according to their capacity to predict the long-form score. The resulting short-forms were then validated against long-form NEMS instruments in 193 outlets, producing comparable results, and a sizeable reduction in time taken to administer the instrument was noted ${ }^{(21)}$.

The present paper reports the results of a comparison between scores obtained using the longer versions of $\operatorname{NEMS}^{(19,20)}$ and the short-form versions ${ }^{(21)}$ on an Australian sample of food outlets. The aim was to critically examine whether important aspects of the food environment known to influence eating behaviour were captured by each instrument, therefore reflecting on the capacity of each tool to assess the healthfulness of the consumer food environment. A further aim was to determine if the shortforms produced comparable scores to the longer versions in an Australian context, thereby demonstrating whether the brief instruments could deliver comparable assessments in a more resource-economical form.

\section{Methods}

\section{Data collection}

Data from three Australian studies of the consumer food environment, conducted in 2014 ${ }^{(22)}, 2016^{(23)}$ and 2018 (RJ Storr, JE Carins \& SR Rundle-Thiele, unpublished results), were used for the current analysis. The studies were conducted in four suburban areas in Brisbane, Australia: one area with high socio-economic status (SES) $2 \mathrm{~km}$ from city; one medium-SES area $6 \mathrm{~km}$ from city; one low-SES area $13 \mathrm{~km}$ from city; and a further low-SES area $50 \mathrm{~km}$ from city ${ }^{(24)}$. The first two suburbs were less culturally diverse (70-80\% Australian by birth, with 50-60\% both parents Australian by birth) than the second two suburbs (50-65\% Australian by birth, with 25-40\% both parents Australian by birth). The study designs were based on the original NEMS studies ${ }^{(19,20,25)}$ and used the validated NEMS-R and NEMS-S following the protocols specified in the NEMS training manual (www.med.upenn.edu/nems). Both instruments were modified slightly for the Australian context by changing some foods to common, but equivalent, leading Australian foods and brands. Modifications were in accordance with the recommendations found on the NEMS website, were pilot-tested prior to the first study and did not disturb the categories or scoring structure of either instrument. During modification, the researchers considered the applicability of the instruments to the Australian context by referring to available literature on Australian dietary practices and purchasing patterns $^{(26,27)}$, finding a high degree of similarity between Australia and the USA. Food outlets within a $1 \mathrm{~km}$ radius of the centre of each suburb, along with all supermarkets within a $2 \mathrm{~km}$ radius, were identified for assessment (because few supermarkets were found within the $1 \mathrm{~km}$ zone). Outlets were sorted into types (stores and restaurants); assessed using the correct NEMS instrument (NEMS$\mathrm{S}$ or NEMS-R) by a team of academics and research students; and from this assessment, scored using the longform scoring method ${ }^{(19,20)}$ and the short-form method ${ }^{(21)}$.

\section{Data analysis}

The original NEMS observational instruments require data for a number of items to be recorded in either yes/no form or count form, or to record detail of an observation (e.g. the price of an item). Although the instruments contain many questions, the score in both long- and short-form versions is derived from fewer findings (e.g. one question may require the price of whole milk to be recorded, and another the price of low-fat milk; whereas points are attributed to the result of whether low-fat milk is priced higher or lower than whole milk). Both the NEMS long-form scoring method ${ }^{(19,20)}$ and the short-form method $^{(21)}$ were examined to identify which items contributed to the score and to appraise the scoring procedure used. This allowed critical consideration of whether each instrument captured important aspects of the food environment known to influence eating behaviour and therefore whether they were robust tools for assessing the healthfulness of the consumer food environment. Long-form scores were calculated using the NEMS protocols found in the training manual and the short-form scores calculated using the method specified ${ }^{(21)}$ to enable 
comparison between the long- and short-forms in an Australian context.

\section{Statistical analyses}

The statistical software package IBM SPSS Statistics version 23 was used for analysis. The statistical analysis sought to determine whether the scores produced by the short-form were comparable to those produced by the long-form. The Wilcoxon matched-pairs signed-rank test was used to compare the long-form and short-form scores for each outlet for the group of restaurants and the group of stores; and for each outlet type within these groups. The Wilcoxon matched-pairs signed-rank test was used to determine whether the score produced by one form of instrument is more often higher, or lower, than the other method. Paired-samples $t$ tests were also conducted to determine the average size of the difference between the instruments. However, paired-samples $t$ tests will not indicate if the scores produced by one method are more frequently higher or lower compared with the other, due to the potential effect of any scores that may be located far from the mean.

\section{Results}

Tables 1 and 2 compare scoring opportunities (as a result of individual items or findings derived from multiple items) for NEMS-R and NEMS-S long-form and short-form versions. This comparison demonstrates which aspects of the consumer food environment are measured by each version of the NEMS (long-form or short-form) when determining the level of support for healthful eating.

NEMS-R long-form captured six consumer-level food environment dimensions, while NEMS-R short-form captured between four and five dimensions, omitting between 17 and $33 \%$ of the consumer-level food environment dimensions previously measured. NEMS-S long-form captured four consumer-level food environment dimensions while NEMS-S short-form captured between one and two dimensions, omitting between 50 and $75 \%$ of the consumer-level food environment dimensions previously measured. The consumer-level food environment dimensions omitted in NEMS-R and NEMS-S are price, quality, placement and facilitators. Specifically, the comparisons in Tables 1 and 2 show that more than half of the scoring opportunities for availability were retained for both instrument types (stores and restaurants); whereas none of the scoring for price was retained. For restaurants, fewer than half of the scoring opportunities for facilitators, barriers, information and promotion were retained. For stores, fewer than half of the scoring opportunities for quality and placement were retained.

A total of 159 outlets were identified for assessment from maps and street directories; however, twelve (8\%) had closed prior to assessment, nine (6\%) store operators declined assessment and three (2\%) outlets were considered unsuitable for assessment (spice store/wedding cake store/bodybuilding supplement store). The studies were conducted in $2014^{(22)}, 2016^{(23)}$ and 2018 (RJ Storr, JE Carins \& SR Rundle-Thiele, unpublished results). The final data set contained twenty-seven sit-down restaurants, twenty-one fast casual restaurants, thirty-four fast-food outlets and eighteen specialty food stores - all scored with the NEMS-R instrument; and fifteen grocery and twenty convenience stores - all scored with the NEMS-S instrument. Distributions of outlet types within each suburb were similar, except that sit-down restaurants were more common in the first (high-SES) suburb and fast-food outlets were more common in the fourth (low-SES) suburb. Each assessment was complete (no missing data). No variety stores were assessed in these studies. Both longform and short-form median and mean scores are shown in Tables 3 and 4, along with the results of statistical testing.

Of the 135 outlets assessed, the short-form score was higher for fifty-two outlets compared with the long-form score; whereas for eighty-one outlets the short-form score was lower than the long-form score. The short-form method produced a statistically significant median increase in score compared with the long-form score for stores ( $z=3 \cdot 441, P=0 \cdot 001$; Wilcoxon's test). For restaurants, on the other hand, the short-form method produced a statistically significant median decrease in score ( $z=5 \cdot 051, P<0.001$; Wilcoxon's test). For this data set, the results indicate that, in general, the short-form gives an inflated score of support for healthful eating compared with the long-form instrument for stores, but a deflated score for restaurants. When examining the data by outlet

Table 1 Nutrition Environment Measures Survey in Restaurants (NEMS-R): long-form and short-form scoring opportunities for aspects of support for healthy eating

\begin{tabular}{|c|c|c|c|c|c|c|c|c|}
\hline Outlet type & & Availability & Price & Facilitators & Barriers & Information* & Promotion* & $\begin{array}{c}\text { Total number of } \\
\text { dimensions measured }\end{array}$ \\
\hline NEMS-R & LF & 10 & 4 & 3 & 3 & 5 & 7 & 6 \\
\hline Sit-down & SF & 7 & 0 & 1 & 2 & 3 & 3 & 5 \\
\hline Fast casual & SF & 8 & 0 & 1 & 1 & 2 & 2 & 5 \\
\hline Fast food & SF & 7 & 0 & 1 & 2 & 5 & 4 & 5 \\
\hline Specialty & SF & 6 & 0 & 0 & 1 & 4 & 2 & 4 \\
\hline
\end{tabular}

LF, long-form; SF, short-form.

${ }^{*}$ NEMS-R original protocol adds information and promotion item scores to form a sub-score for information. 
Table 2 Nutrition Environment Measures Survey in Stores (NEMS-S): long-form and short-form scoring opportunities for aspects of support for healthy eating

\begin{tabular}{lcccccc}
\hline Outlet type & & Availability $^{*}$ & Price & Quality & Placement* $^{*}$ & $\begin{array}{c}\text { Total number of } \\
\text { dimensions measured }\end{array}$ \\
\hline NEMS-S & LF & 13 & 10 & 2 & 1 & 4 \\
Grocery & SF & 7 & 0 & 1 & 0 & 2 \\
Convenience & SF & 9 & 0 & 1 & 0 & 2 \\
Variety & SF & 7 & 0 & 0 & 0 & 1 \\
\hline
\end{tabular}

LF, long-form; SF, short-form.

*NEMS-S original protocol adds availability and placement item scores to form a sub-score for availability.

Table 3 Scores determined by the NEMS-R ${ }^{(19,20)}$ (long-form) and Reduced Item Audit Tool ${ }^{(21)}$ (short-form) for 100 restaurants representing four suburban areas in Brisbane, Australia $(2014,2016,2018)$

\begin{tabular}{|c|c|c|c|c|c|c|c|c|c|c|c|c|c|c|c|}
\hline \multirow[b]{3}{*}{ Outlet type } & \multirow[b]{3}{*}{$n$} & \multicolumn{14}{|c|}{ Survey scores } \\
\hline & & \multicolumn{2}{|r|}{ LF } & \multicolumn{2}{|c|}{ SF } & \multirow{2}{*}{$\begin{array}{c}\text { SF score } \\
\text { higher }\end{array}$} & \multirow{2}{*}{$\begin{array}{l}\text { SF score } \\
\text { lower }\end{array}$} & \multirow[b]{2}{*}{$z$} & \multirow{2}{*}{$\begin{array}{c}P \\
\text { value }\end{array}$} & \multicolumn{2}{|c|}{ LF } & \multicolumn{2}{|c|}{ SF } & \multirow[b]{2}{*}{$t$} & \multirow{2}{*}{$\begin{array}{c}P \\
\text { value }\end{array}$} \\
\hline & & Median & P25-P75 & Median & P25-P75 & & & & & Mean & SD & Mean & SD & & \\
\hline Sit-down & 27 & $12 \cdot 0$ & $6 \cdot 0-22 \cdot 0$ & $8 \cdot 7$ & $4 \cdot 9-16 \cdot 6$ & 8 & 19 & 1.946 & 0.052 & $14 \cdot 4$ & $13 \cdot 2$ & $12 \cdot 9$ & 11.2 & 1.826 & 0.079 \\
\hline Fast casual & 21 & 9.0 & $4 \cdot 0-13 \cdot 5$ & $7 \cdot 3$ & $4.7-10.3$ & 7 & 14 & 1.373 & 0.170 & 9.0 & 6.9 & 7.5 & $4 \cdot 1$ & 1.431 & 0.168 \\
\hline Fast food & 34 & $13 \cdot 0$ & $5 \cdot 0-25 \cdot 3$ & 11.0 & $3.4-22 \cdot 7$ & 10 & 24 & $3 \cdot 120$ & 0.002 & $15 \cdot 6$ & $14 \cdot 6$ & $12 \cdot 7$ & $12 \cdot 3$ & 3.182 & 0.003 \\
\hline Specialty & 18 & $17 \cdot 0$ & $11 \cdot 3-29 \cdot 3$ & $10 \cdot 8$ & $8 \cdot 4-17.5$ & 1 & 17 & 3.376 & 0.001 & $19 \cdot 3$ & $10 \cdot 8$ & $12 \cdot 0$ & $5 \cdot 5$ & 5.037 & $<0.001$ \\
\hline
\end{tabular}

NEMS-R, Nutrition Environment Measures Survey in Restaurants; LF, long-form; SF, short-form; P25, 25th percentile; P75, 75th percentile.

Table 4 Scores determined by the NEMS-S ${ }^{(19,20)}$ (long-form) and Reduced Item Audit Tool ${ }^{(21)}$ (short-form) for thirty-five stores representing four suburban areas in Brisbane, Australia $(2014,2016,2018)$

\begin{tabular}{|c|c|c|c|c|c|c|c|c|c|c|c|c|c|c|c|}
\hline \multirow[b]{3}{*}{ Outlet type } & \multirow[b]{3}{*}{$n$} & \multicolumn{14}{|c|}{ Survey scores } \\
\hline & & \multicolumn{2}{|r|}{ LF } & \multicolumn{2}{|r|}{ SF } & \multirow{2}{*}{$\begin{array}{c}\text { SF score } \\
\text { higher }\end{array}$} & \multirow{2}{*}{$\begin{array}{l}\text { SF score } \\
\text { lower }\end{array}$} & \multirow[b]{2}{*}{$z$} & \multirow{2}{*}{$\begin{array}{c}P \\
\text { value }\end{array}$} & \multicolumn{2}{|c|}{ LF } & \multicolumn{2}{|c|}{ SF } & \multirow[b]{2}{*}{$t$} & \multirow{2}{*}{$\begin{array}{c}P \\
\text { value }\end{array}$} \\
\hline & & Median & P25-P75 & Median & P25-P75 & & & & & Mean & SD & Mean & SD & & \\
\hline Grocery & 15 & $30 \cdot 8$ & $30 \cdot 0-37.0$ & $30 \cdot 0$ & $27 \cdot 0-33 \cdot 0$ & 11 & 2 & $2 \cdot 412$ & 0.016 & 32.3 & $5 \cdot 1$ & 29.6 & 4.4 & $-3 \cdot 108$ & 0.008 \\
\hline Convenience & 20 & $15 \cdot 2$ & $8.6-19.6$ & 10.5 & $7 \cdot 3-14.5$ & 15 & 5 & 2.465 & 0.014 & $14 \cdot 2$ & 5.5 & 11.6 & $5 \cdot 1$ & -2.680 & 0.015 \\
\hline
\end{tabular}

NEMS-R, Nutrition Environment Measures Survey in Stores; LF, long-form; SF, short-form; P25, 25th percentile; P75, 75th percentile.

type, these patterns were consistent within store types, with the short-form producing a statistically significant median increase in score for grocery and convenience store categories. However, the short-form produced a significant median decrease in the fast-food and specialty restaurant categories only, with differences in the sit-down and fast casual categories failing to reach significance. Calculations indicate that a minimum sample size of ninety-four provides $80 \%$ power at $\alpha=0.05$ level to detect a small effect $(d=0 \cdot 3)$ using the Wilcoxon's test. The mean effect size observed in this sample of 135 outlets was 1.6 points with an SD of $5 \cdot 7$ (equivalent to $d=0.3$ ).

\section{Discussion}

Researchers seek valid and reliable tools and techniques to provide robust information for research and programmes while keeping time and costs manageable in resourceconstrained situations. Reducing the length of existing (long-form) tools is one pathway towards more cost- efficient measurement. Taking this approach, Partington et $a{ }^{(21)}$ found comparable results when measuring support for healthful eating with short-form versions of NEMS in a US sample of stores and eating outlets.

The current study undertook a critical comparison of long- and short-form measures, drawing on available data ${ }^{(22,23)}$; RJ Storr, JE Carins \& SR Rundle-Thiele, unpublished results) scored using the long-form NEMS scoring protocols and following short-form analysis guidelines reported in Partington et al. ${ }^{(21)}$. On an Australian sample of stores and restaurants comparable results were not identified: in general, the short-form delivered an inflated score compared with the long-form version for stores, and a deflated score for restaurants. The raw scores varied by as much as double (for stores) and half (for restaurants). This indicates that the pattern of contribution made by individual items to the overall score was different in this Australian sample compared with the US sample ${ }^{(21)}$. For stores, the longform score is derived from thirteen availability items, ten price items, two quality items and one placement item; whereas the short-form score is obtained from availability 
items and one quality item. This suggests that dimensions of price, quality and placement were stronger and more negative influences on support for healthful eating in these Australian studies. For restaurants, interpretation is not as straightforward. For these outlets, the long-form score is derived from ten availability items, four price items, three items for facilitators and three for barriers, five information items and seven promotion items. The spread of items in the short-form is similar, except that availability items are mostly retained and all price items are removed. A cautious interpretation of the deflated short-form scores for restaurants could be that pricing was a stronger, more positive influence - or that availability was a weaker, more negative influence - on support for healthful eating in the current study. Nevertheless, the current results suggest that more testing of the short-form measure ${ }^{(21)}$ will be required to obtain a short-form measure that delivers comparable results.

Face validity involves making an assessment about the items used to measure a construct ${ }^{(28)}$ and is deemed to occur when 'a scale's content logically appears to reflect what was (initially) intended to be measured' ${ }^{(29)}$. Many features of the consumer food environment influence eating behaviour, which makes it difficult to measure them all within an instrument and difficult to understand what should be included in a food environment construct ${ }^{(4)}$. Taking a critical stance, the present study notes that the long-form NEMS-R captures six consumer-level food dimensions while the long-form NEMS-S captures four dimensions. Both long-form measures omit dimensions such as access, affordability, variety and freshness, all of which enhance healthy eating ${ }^{(6,30,31)}$. The data-driven approach taken to derive the short-form measure ${ }^{(21)}$ omitted many environmental dimensions captured in the original long-form measure, reducing it to primarily a measure of availability. Availability of healthful food products which remained a cornerstone of the short-form measure is essential for healthy eating, but many other factors contribute and need to be considered for inclusion in measurement tools like $\operatorname{NEMS}^{(9)}$. This ensures these tools can be used to benchmark support for healthy eating within food environments and to measure the progress of initiatives designed to improve food environments.

Consider that products are placed on shelves deliberately to increase prominence and salience ${ }^{(32,33)}$, which increases the likelihood of their purchase. Experimental studies have recently shown the effect of modifying shelf placement to increase sales of healthful dairy products ${ }^{(34)}$. Research has shown that although unhealthy options dominate shelf space in many stores, this varies across stores $^{(35)}$, making prominence of placement an important aspect to consider in food environment measurement tools. Placement was minimally measured in the long-form version of NEMS and was absent from the short-form version.

Price is considered one of the strongest marketing factors predicting energy intake ${ }^{(36)}$. Studies have shown that price increases for supermarket goods can drive down purchases ${ }^{(37)}$ and price discounts for healthy foods can stimulate purchases ${ }^{(38)}$, suggesting this dimension of the food environment remains an important feature to measure. A large randomised trial found price discounts for healthier products had a significant positive effect on purchases, which lasted beyond the period of discounting. In the same trial, carefully tailored nutrition education had no effect on food purchases ${ }^{(39)}$. No pricing elements were included in the short-form NEMS version for either stores or restaurants. While a balance must be struck between the need for information and whether the level of detail is easily obtained, the comparative measures included in the long-form versions of NEMS indicated whether healthier alternatives items were sold at a premium or whether discounting favoured overeating. As price is such a strong influence on behaviour ${ }^{(36-38)}$, consideration should be given to retaining a measure of price in a shortened version of NEMS.

Facilitators and barriers to healthy eating exist in many eating outlets, especially restaurants and fast-food outlets, and often influence diners without awareness. Many eating decisions occur at a low level of consciousness ${ }^{(40)}$, with factors such as portion size, presentation, free refills, supply and proximity of foods all affecting consumption $^{(41)}$. Continued or re-emphasised measurement of facilitators and barriers in tools like NEMS is advisable. Nutrition information supports those who are consciously and deliberately trying to eat better, but can take skill and effort to interpret, and hence often does not have the desired intended effect of reducing energy intake ${ }^{(42)}$. The demand for foods eaten away from home is sensitive to price change ${ }^{(37)}$ and is encouraged by bundling (or bulk discounting) as consumers consider value for money to be one of the most important factors influencing their purchase $^{(43)}$. Further to this, promotion, advertising and media are important aspects to measure in the food environment, both in supermarkets and food-service outlets. Media and advertising for food products exceeds \$AUD 400 million in Australia $^{(44)}$ and these influences are widely acknowledged to be pervasive ${ }^{(36)}$.

Taken together, discrepancies on dimensions scored and the absence of some dimensions in the short-form measures suggest further research is warranted before conclusions can be drawn on whether the short-form measures adequately capture aspects of the consumer food environment known to affect healthy eating in this Australian context.

Availability of healthful alternatives is more heavily represented in the short-forms, with inflated scores for these dimensions observed in the current study at the expense or complete absence of other dimensions, namely quality, placement, information, promotion, facilitators, barriers and price. Other efforts to develop shortform measures of outlet healthfulness have also focused only on availability of foods ${ }^{(45)}$, thereby removing the 
ability to assess other aspects of the food environment known to affect consumer purchase and consumption of foods.

Partington et al. ${ }^{(21)}$ acknowledge that shortening the form increases the risk that important information may be lost, and the present paper shares this concern. Future iterations of the NEMS should bear in mind the brevity of the instrument, but also aim to include important factors known to influence consumers so that opportunities for intervention can be identified and mapped in communities. Consideration of face validity in addition to criterion validity is recommended.

\section{Limitations and future research}

The current sample was drawn from four suburbs in Australia and therefore does not cover the range or variety of food outlets present nationally, with some outlet types not represented in the sample (variety stores and specialty stores were absent). The sample size is more than twothirds that used previously to validate the short-form ${ }^{(21)}$ and findings require further verification with a larger, more diverse, sample size. We estimate our study was sufficiently powered to detect small differences across the entire sample, but only larger differences would have been observed as significant in the smaller subgroups of outlets. Assessment of a larger sample of outlets from other areas containing all outlet types with NEMS short- and long-forms represents an opportunity for future research before definitive conclusions are drawn. Further research is recommended to develop a measure of the consumerlevel food environment capturing additional dimensions, including affordability, access, freshness and variety. Continued emphasis on developing short-form NEMS-R and NEMS-S measures is recommended to enable costeffective measurement. Alternative analytical methods are recommended to develop a short-form measure. For example, Mokken's ${ }^{(46)}$ model of monotone homogeneity is recommended to investigate item strength. The model of monotone homogeneity estimates the extent to which pairs of items approximate an ideal, permitting an assessment of whether they can be explained by the same underlying trait ${ }^{(47)}$. That said, the number of dimensions and the nature of measurement used in an assessment of the food environment ultimately depend on the objectives of the research at hand and must balance the need to capture important information while keeping costs manageable. Finally, the current study was centred at the consumer level. critically examining NEMS-R and NEMS-S short- and long-form measures. Additional opportunities exist to extend understanding to social and community levels given NEMS measures could potentially accommodate these levels. For example, the number of stores within a given radius is captured in NEMS measurement, permitting the community-level dimension of store types within a suburb to be determined. Future research could employ a mixed-method approach to develop new or alternative versions of NEMS that tap into emerging aspects of the consumer food environment known to influence eating behaviour. The development and validation of new or revised instruments would provide a greater suite of tools to enable researchers to select the measures most suited to their research objectives.

\section{Conclusions}

An initial examination of short-form versions of NEMS in an Australian sample did not produce comparable results with the long-forms. Notably, an inflated score for stores using the NEMS-S short-form, and a deflated score for restaurants using the NEMS-R short-form, were evident. Balance is needed to produce tools that provide robust insight and do not take long to administer. It would be desirable to ensure that future versions of food environment measurement tools like NEMS retain items that measure pricing; cover facilitators of and barriers to healthy eating well; and are extended to include the advertising and media environment.

\section{Acknowledgements}

Financial support: This research was conducted under a collaborative research agreement between Griffith University and the Defence Science and Technology Group (DST Group). The authors would like to acknowledge DST Group for providing financial support for this study (via a student scholarship). Conflict of interest: None. Authorship: J.E.C. led the study design and execution; analysis and interpretation; and authoring of the manuscript. S.R.-T. contributed to study design; interpretation; and authorship of the manuscript. R.J.S. contributed to study design and execution; analysis; and critical reviewing of the final manuscript. Ethics of buman subject participation: The study protocol was considered by the GU Ethics Committee and assessed as not requiring formal ethical review.

\section{References}

1. World Health Organization (2003) Diet, Nutrition and the Prevention of Chronic Disease. Joint WHO/FAO Expert Consultation. WHO Technical Report Series no. 916. Geneva: WHO.

2. Ng M, Fleming T, Robinson $\mathrm{M}$ et al. (2014) Global, regional, and national prevalence of overweight and obesity in children and adults during 1980-2013: a systematic analysis for the Global Burden of Disease Study 2013. Lancet 384, 766-781.

3. Vandevijvere S \& Tseng M (2013) Towards comprehensive global monitoring of food environments and policies to reduce diet-related non-communicable diseases. Public Health Nutr 16, 2101-2104.

4. Lytle LA \& Sokol RL (2017) Measures of the food environment: a systematic review of the field, 2007-2015. Health Place 44, 18-34. 
5. Ohri-Vachaspati P \& Leviton LC (2010) Measuring food environments: a guide to available instruments. Am J Health Promot 24, 410-426.

6. Caspi CE, Sorensen G, Subramanian SV et al. (2012) The local food environment and diet: a systematic review. Health Place 18, 1172-1187.

7. Glanz K, Sallis JF, Saelens BE et al. (2005) Healthy nutrition environments: concepts and measures. Am J Health Promot 19, 330-333.

8. Lytle LA (2009) Measuring the food environment: state of the science. Am J Prev Med 36, 4 Suppl., S134-S144.

9. Glanz K, Johnson L, Yaroch AL et al. (2016) Measures of retail food store environments and sales: review and implications for healthy eating initiatives. J Nutr Educ Behav 48, 280-288.

10. Pereira RF, Sidebottom AC, Boucher JL et al. (2014) Assessing the food environment of a rural community: baseline findings from the Heart of New Ulm Project, Minnesota, 2010-2011. Prev Chronic Dis 11, E36.

11. Cannuscio CC, Tappe K, Hillier A et al. (2013) Urban food environments and residents' shopping behaviors. Am J Prev Med 45, 606-614.

12. Gustafson AA, Sharkey J, Samuel-Hodge CD et al. (2011) Perceived and objective measures of the food store environment and the association with weight and diet among low-income women in North Carolina. Public Health Nutr 14, 1032-1038.

13. Lesser LI, Hunnes DE, Reyes P et al. (2012) Assessment of food offerings and marketing strategies in the food-service venues at California children's hospitals. Acad Pediatr 12, 62-67.

14. Horacek TM, Erdman MB, Byrd-Bredbenner C et al. (2013) Assessment of the dining environment on and near the campuses of fifteen post-secondary institutions. Public Health Nutr 16, 1186-1196.

15. Horacek TM, Erdman MB, Reznar MM et al. (2013) Evaluation of the food store environment on and near the campus of 15 postsecondary institutions. Am J Health Promot 27, e81-e90.

16. Olstad DL, Raine KD \& McCargar LJ (2012) Adopting and implementing nutrition guidelines in recreational facilities: public and private sector roles. A multiple case study. BMC Public Health 12, 376.

17. Canto SD, Engler-Stringer R \& Muhajarine N (2015) Characterizing Saskatoon's food environment: a neighbourhoodlevel analysis of in-store fruit and vegetable access. Can J Urban Res 24, 62-77.

18. Khan S, Calloway SA, Maida TI et al. (2012) Dietary and built environment assessment in a Latino community. $A m J$ Health Educ 43, 74-82.

19. Glanz K, Sallis JF, Saelens BE et al. (2007) Nutrition Environment Measures Survey in stores (NEMS-S): development and evaluation. Am J Prev Med 32, 282-289.

20. Saelens BE, Glanz K, Sallis JF et al. (2007) Nutrition Environment Measures Study in restaurants (NEMS-R): development and evaluation. Am J Prev Med 32, 273-281.

21. Partington SN, Menzies TJ, Colburn TA et al. (2015) Reduced-item food audits based on the nutrition environment measures surveys. Am J Prev Med 49, e23-e33.

22. Carins JE \& Rundle-Thiele SR (2014) Fighting to eat healthfully: measurements of the military food environment. J Soc Mark 4, 223-239.

23. Storr RJ (2016) Comparison of the nutrition environment in advantaged and disadvantaged neighbourhoods in Brisbane. Honours Dissertation, Griffith University.

24. Australian Bureau of Statistics (2011) ABS Social Economic Indexes for Areas. http://www.abs.gov.au/websitedbs/ censushome.nsf/home/seifa (accessed January 2018).
25. Frank L, Glanz K, McCarron M et al. (2006) The spatial distribution of food outlet type and quality around in differing built environment and demographic contexts. Berk Plann J 19, 79-95.

26. McLennan W \& Podger AS (1999) National Nutrition Survey: Foods Eaten: Australia 1995. Canberra: Australian Bureau of Statistics.

27. Euromonitor International (2013) Brand Shares. Euromonitor Passport Database. www.portal.euromonitor.com (accessed September 2013).

28. Rossiter JR (2002) The C-OAR-SE procedure for scale development in marketing. Int J Res Mark 19, 305-335.

29. Zikmund W, Babin B, Carr J et al. (2013) Measuring and scaling concepts. In Business Research Methods, pp. 289-309. Mason, OH: South-Western Cengage Learning.

30. Lee JH, Ralston RA \& Truby H (2011) Influence of food cost on diet quality and risk factors for chronic disease: a systematic review. Nutr Diet 68, 248-261.

31. Cummins S, Petticrew M, Sparks L et al. (2005) Large scale food retail interventions and diet. BMJ 330, 683-684.

32. Dreze X, Hoch SJ \& Purk ME (1994) Shelf management and space elasticity. J Retail 70, 301-326.

33. Campo K, Gijsbrechts E \& Nisol P (2003) The impact of retailer stockouts on whether, how much and what to buy. Int J Res Mark 20, 273-286.

34. Adam A, Jensen JD, Sommer I et al. (2017) Does shelf space management intervention have an effect on calorie turnover at supermarkets? J Retail Consum Serv 34, 311-318.

35. Farley TA, Rice J, Bodor JN et al. (2009) Measuring the food environment: shelf space of fruits, vegetables, and snack foods in stores. J Urban Health 86, 672-682.

36. Chandon P \& Wansink B (2012) Does food marketing need to make us fat? Nutr Rev 70, 571-593.

37. Andreyeva T, Long MW \& Brownell KD (2010) The impact of food prices on consumption: a systematic review of research on the price elasticity of demand for food. $A m J$ Public Health 100, 216-222.

38. Waterlander WE, Steenhuis IHM, de Boer MR et al. (2012) The effects of a $25 \%$ discount on fruits and vegetables: results of a randomized trial in a three-dimensional webbased supermarket. Int J Behav Nutr Phys Act 9, 11.

39. Mhurchu CN, Blakely T, Jiang Y et al. (2010) Effects of price discounts and tailored nutrition education on supermarket purchases: a randomized controlled trial. Am J Clin Nutr 91, 736-747.

40. Cohen DA \& Farley TA (2008) Eating as an automatic behavior. Prev Chronic Dis 5, A23.

41. Wansink B \& Sobal J (2007) Mindless eating: the 200 daily food decisions we overlook. Environ Behav 39, 106-123.

42. Swartz JJ, Braxton D \& Viera AJ (2011) Calorie menu labeling on quick-service restaurant menus: an updated systematic review of the literature. Int J Behav Nutr Phys Act $\mathbf{8}, 135$.

43. Vermeer WM, Steenhuis IH \& Seidell JC (2010) Portion size: a qualitative study of consumers' attitudes toward point-ofpurchase interventions aimed at portion size. Health Educ Res 25, 109-120.

44. Jolly R (2013) Marketing Obesity? Junk Food, Advertising and Kids. Canberra: Parliament of Australia.

45. DeWeese RS, Todd M, Karpyn A et al. (2016) Short-form audit instrument for assessing corner store healthfulness. Am J Health Promot 32, 224-232.

46. Mokken RJ (1971) A Theory and Procedure of Scale Analysis: With Applications in Political Research. The Hague: Mouton.

47. Sijtsma K \& Molenaar IW (2002) Introduction to Nonparametric Item Response Theory. Thousand Oaks, CA: SAGE Publications, Inc. 